\title{
Timely Diagnosis of Heparin-Indused Thrombocytopenia: The Proposal for a Right Choice
}

\author{
D D’Arco ${ }^{1 * \#}$, A Mangino ${ }^{1 * \#}$, N Pepe $^{1}$, V Maddaloni ${ }^{1}$, M Coppola ${ }^{1}$, L Atripaldi $^{3}$, U Atripaldi ${ }^{3}$, A Sabatino ${ }^{1}$, R \\ Sabatino $^{1}$, T Di Matola ${ }^{1}$ and L Atripaldi ${ }^{1,2}$ \\ ${ }^{1}$ Chemical biochemistry Unit, AORN dei Colli, Monaldi Hospital, Italy \\ ${ }^{2}$ Microbiology and Virology Unit, AORN dei Colli, Cotugno Hospital, Italy \\ ${ }^{3}$ University of Campania Luigi Vanitelli, Italy \\ ${ }^{\#}$ These authors contributed equally to this work
}

*Corresponding author: D’Arco D, Chemical biochemistry Unit, AORN dei Colli, Monaldi Hospital, VL Bianchi, 80131 Naples, Italy

A Mangino, Chemical biochemistry Unit, AORN dei Colli, Monaldi Hospital, VL Bianchi, 80131 Naples, Italy

\section{ARTICLE INFO}

Received: 幽 February 11, 2020

Published: 业 February 19, 2020

Citation: D’Arco D, A Mangino, N Pepe, V Maddaloni, M Coppola, L Atripaldi, U Atripaldi, A Sabatino, R Sabatino, T Di Matola, L Atripaldi. Timely Diagnosis of Heparin-Indused Thrombocytopenia: The Proposal for a Right Choice. Biomed J Sci \& Tech Res 25(5)-2020. BJSTR. MS.ID.004256.

Keywords: Heparin Induced Thrombocytopenia; 4T Score; AbHIT; Chemiluminescence Immunological Test

\section{ABSTRACT}

Heparin Induced Thrombocytopenia (HIT) is an acute transient prothrombotic complication combined with thrombocytopenia. This pathology is characterized by the presence of antibodies (AbHIT) which bind PF4-Hep complexes and activate platelets. AbHITs are present in the bloodstream since the onset of this pathology and disappear completely after about 100 days [1]. The PF4/heparin/antibody complex binds both the membrane of the circulating platelets and the endothelial cells resulting in platelet activation; the further release of PF4 and endothelial damage induce, in turn, the release of tissue factor and the monocyte activation. This mechanism is a real positive feedback and involves a massive thrombin formation that causes a fall in the platelet count of about $20-30 \%$, compared to their basal level, often associated with complications such as venous thrombosis, arterial thrombosis, stroke and myocardial infarction [2]. The purpose of our study was to monitor the epidemiological course of the HIT, to observe the differences between the several tests used for its diagnosis, to study the trend of some biological variables and their correlation with HIT, and to understand the degree of knowledge of healthcare personnel about the group of rare diseases to which the HIT belongs.

\section{Introduction}

Heparin Induced Thrombocytopenia (HIT) is a rare disease first described in the 1950s [3].It is a pro-thrombotic induced drug reaction caused by the presence of antibodies (AbHIT) which bind the "Platelet Factor 4 (PF4) Heparin (Hep)" complex and activate platelets $[2,4]$. When this pathology occurs, there is a decrease in the platelet count $\left(<150000 / \mathrm{mm}^{3}\right)$ and a high risk of developing thrombotic phenomena. The diagnosis of HIT is carried out through a collection of clinical data (4T Score) associated with immunological and functional tests $[2,4,5]$. The $4 \mathrm{~T}$ Score (Thrombocytopenia - Timing of platelet count fall- Thrombosis or other sequelae - Other causes for thrombocytopenia) summarizes the identification conditions of HIT and therefore represents a valid clinical support to exclude this pathology in patients who develop thrombocytopenia in the hospital setting. It is usually used as a first level test and it is therefore seen as a screening test; in fact, it considers the use of a 'scoring system' to stratify patients with thrombocytopenia who have been treated with heparin.

It has been shown by many study groups that the $4 \mathrm{~T}$ score has an efficiency of $94 \%$ and that its positive predictive value depends mainly on the competence and experience of the clinician who is examining the potentially positive patient [6]. The second most important tests are the platelet activation assays and the immunological tests carried out to detect the presence of antibodies (AbHIT) in the blood of patients potentially affected by this pathology. A greater understanding of HIT could lead to increasingly specific approaches preventing the formation of complex-mediated thrombi and favoring a less use of systemic anticoagulant therapy. For this reason, a prospective study was undertaken in the UOC 
of Clinical Biochemistry of the Monaldi Hospital (AORN deiColli) which focuses on both the diagnosis and the epidemiological course of HIT and the innovative and automated method mentioned above.

\section{Materials and Methods}

This study focuses on the most common tests useful for HIT diagnosis:

1. $4 \mathrm{~T}$ Score

2. Immunological tests

3. Quantitative assessments and trends of some biological variables

The 4T score, through a "scoring system", is used to stratify patients with thrombocytopenia treated with heparin. Patients with a low score (0-3 points) belong to the "low risk" category and have a very good chance of not being affected by HIT. Patients with a higher score belong to the "medium-high risk" category and the higher the score the greater the probability of being affected by HIT. In these cases, however, the diagnosis of HIT must be confirmed with further tests. The immunological tests allow to detect the presence of antibodies $(\mathrm{Ab})$ or antigens $(\mathrm{Ag})$ in circulating blood. The fully automated chemiluminescence immunological test, carried out with the ACL AcuStar (Werfen), allows the quantitative determination of the anti-PF4/Hep AbHIT in plasma; this test uses magnetic beads adhered with PF4 bound to polyvinyl sulfonate for the direct research of AbHIT in patients with suspected HIT. After an incubation, a magnetic separation and a washing step, a solution containing anti-IgG antibodies is added which capture, if they are present, the anti-PF4/Hep IgG antibodies; later it occurs a second incubation, magnetic separation and washing step and, after, the addition of the reagents which lead to the chemiluminescence reaction and, therefore, the quantitative determination. The emitted light is measured by the optical system of the AclAcuStar.

The detected biological variables are:

1. Platelets: analyzed through the blood count test.

2. D-Dimer: quantized with an automatic lateximmunoassay.

3. Fibrinogen: quantized with the Clauss method.

4. Immunoglobulins: detected with nephelometry.

\section{Results}

This study was conducted on 144 patients, of whom 127 are patients with clinical suspicion of HIT and 17 are control patients. The 17 control patients group consists of 8 healthy volunteers, 4 patients with thrombocytopenia resulting from other causes and 5 patients under heparin treatment for more than 7 days without thrombocytopenia. The group of 127 potentially positive patients is made up of subjects who come from a large hospitalized population (orthopedics, cardiac surgery and intensive care) (Figure 1). We found 16 HIT positive patients and 1 borderline patient; among the 16 positive patients, we have choosen some example cases to show the trend of several biological variables such as: Platelets (PLT), Fibrinogen (QFA), D-Dimer (DD), Antibody Classes (IgG, IgA and (gM).

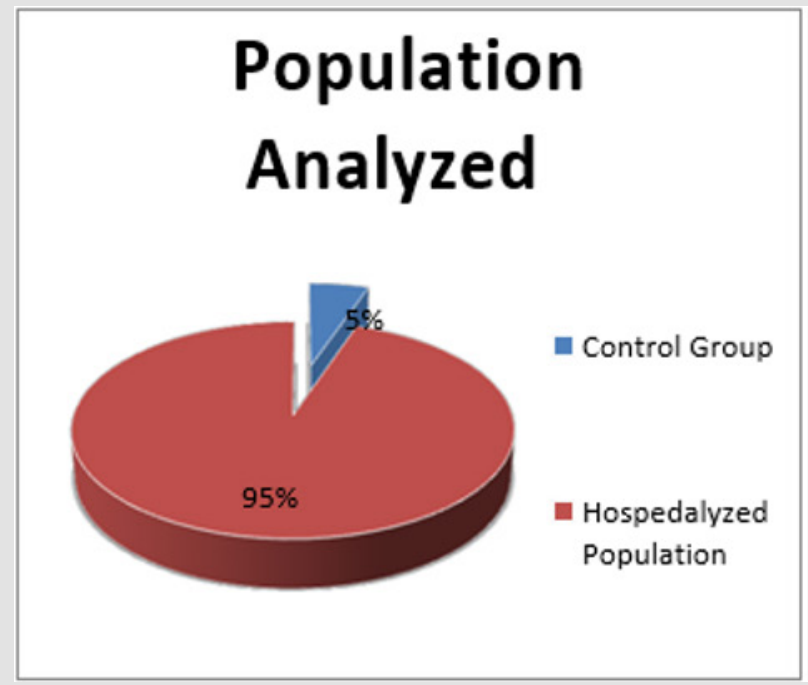

\section{Hospedalysed Population}
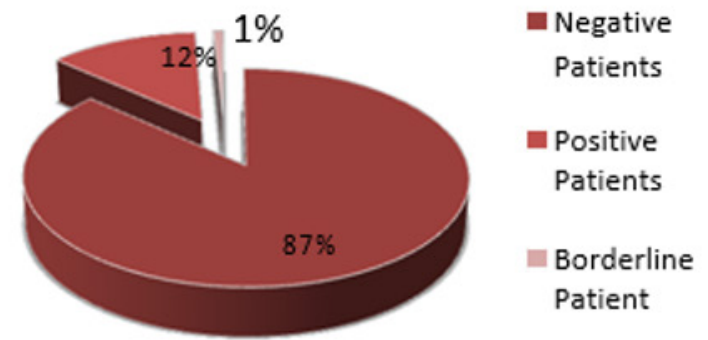

Figure 1: Percentages of the Population Analysed and Hospitalized Population.

After replacing the heparin (ENF or EBPM) with a new anticoagulant drug, we have observed:

1. a platelet value recovery within a few days of the switch.
2. almost stable QFA and DD values.

3. a return to normal values of the antibody classes (IgG, IgA and $\operatorname{IgM}$ ) despite the increase in IgG expression found during the period in which HIT positivity could be detected (Figure 2). 


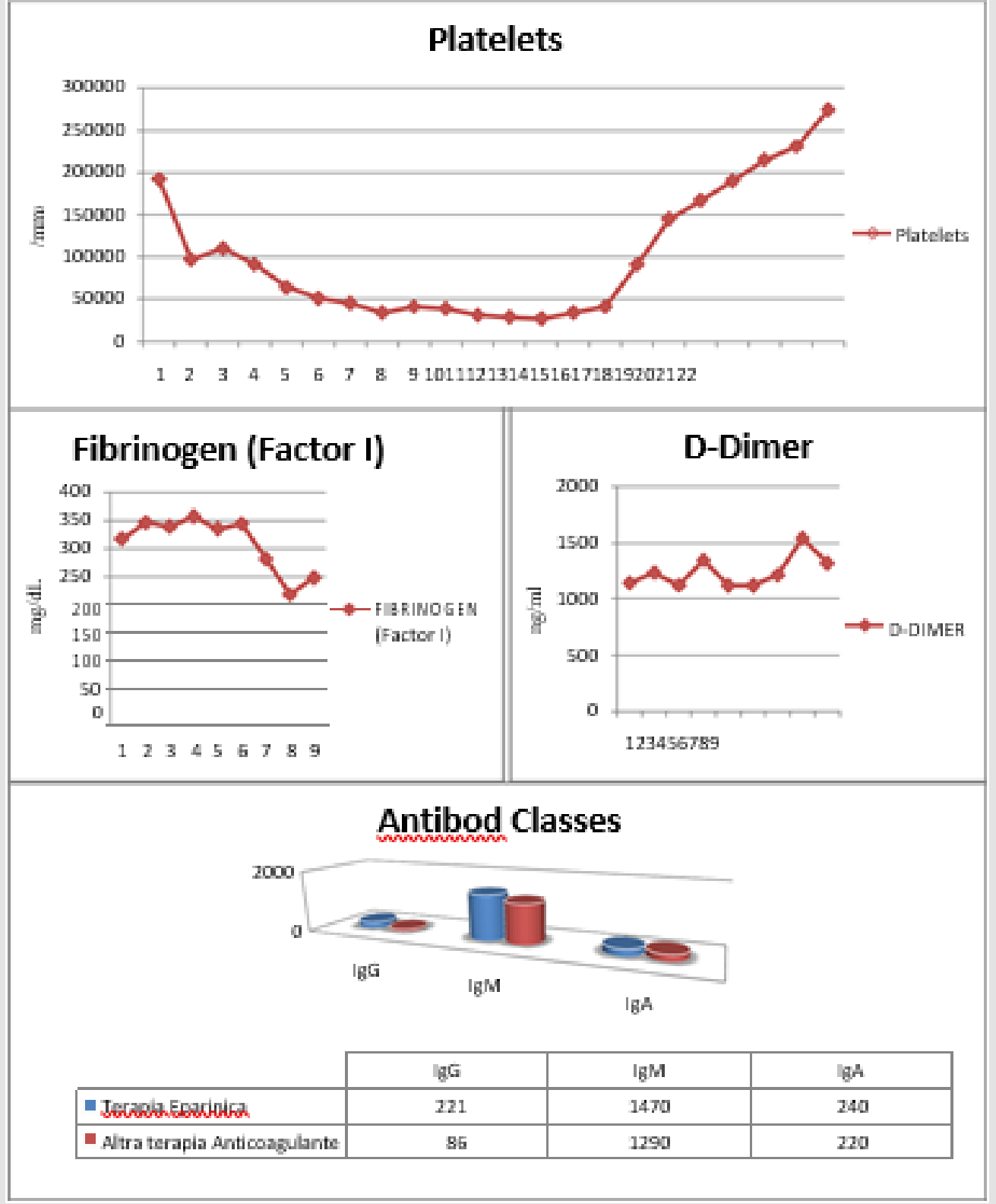

Figure 2: Trend of some biologicalvariables (PLT, QFA, DD, AntibodyClasses) in a patientexample.

\section{Discussion and Conclusions}

The results of this study show that the chemiluminescence immunological test, useful for the detection of AbHIT, has a higher precision and reliability than the Score of the $4 \mathrm{~T}$. However, a continuous professional staff training on the group of rare diseases, to which heparin-induced thrombocytopenia belongs, is definitely fundamental. In addition, through this prospective study, it has been possible to draw greater attention to similar cases and to urge and to encourage clinicians to carry out the necessary checks in order to guarantee both early diagnosis and better treatment of potentially positive patients, and, therefore, the quality of care of HIT patients in the AORN deiColli (Monaldi - Cotugno - C.T.O.) has been positively influenced.

\section{References}

1. Warkentin TE, Kelton JG (2001) Temporal aspects of heparin-induced thrombocytopenia. N Engl J Med 344(17): 1286-1292.

2. Warkentin TE Heparin (2003) Induced thrombocytopenia: pathogenesis and management. Br J Haematol 121(4): 535-555.

3. Wiesmann RE, Tobin RW (1958) Arterial embolism occuring during systemic heparin therapy . Arch Surg. 76(2): 219-225.

4. Chong BH. Heparin (2003) Induced thrombocytopenia. J Thromb Haemost 1(7): 1471-1478.

5. Warkentin TE, Hayward CP, Smith CA, Kelly PM, Kelton JG (1992) Determinants of donor platelet variability when testing for heparininduced thrombocytopenia. J Lab Clin.Med. 120(3):371-379.

6. Klein HG, Bell WR (1974) Disseminated intravascular coagulation during heparin therapy. Ann Intern Med. 80(4): 477-81. 
ISSN: 2574-1241

DOI: 10.26717/BJSTR.2020.25.004256

D’Arco D, A Mangino. Biomed J Sci \& Tech Res

(c) (P) This work is licensed under Creative

Submission Link: https://biomedres.us/submit-manuscript.php

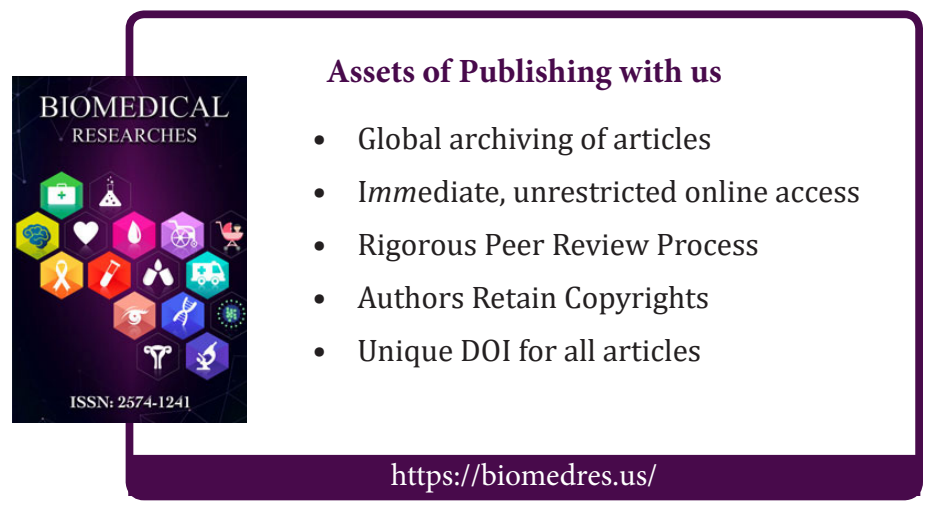

